\title{
"Inflammaging" as a Druggable Target: A Senescence-Associated Secretory Phenotype-Centered View of Type 2 Diabetes
}

\author{
Francesco Prattichizzo, ${ }^{1,2}$ Valeria De Nigris, ${ }^{1}$ Lucia La Sala, ${ }^{3}$ \\ Antonio Domenico Procopio, ${ }^{2,4}$ Fabiola Olivieri, ${ }^{2,4}$ and Antonio Ceriello ${ }^{1,3}$ \\ ${ }^{1}$ August Pi i Sunyer Biomedical Research Institute (IDIBAPS) and Centre of Biomedical Investigation on Diabetes and \\ Associated Metabolic Disorders Network (CIBERDEM), 08036 Barcelona, Spain \\ ${ }^{2}$ Department of Clinical and Molecular Sciences, DISCLIMO, Università Politecnica delle Marche, 60126 Ancona, Italy \\ ${ }^{3}$ IRCCS Multimedica Group, 60126 Sesto San Giovanni, Italy \\ ${ }^{4}$ Center of Clinical Pathology and Innovative Therapy, Italian National Research Center on Aging (INRCA-IRCCS), \\ 60121 Ancona, Italy \\ Correspondence should be addressed to Francesco Prattichizzo; f.prattichizzo@univpm.it
}

Received 26 February 2016; Accepted 9 May 2016

Academic Editor: Mark J. Crabtree

Copyright (C) 2016 Francesco Prattichizzo et al. This is an open access article distributed under the Creative Commons Attribution License, which permits unrestricted use, distribution, and reproduction in any medium, provided the original work is properly cited.

\begin{abstract}
Aging is a complex phenomenon driven by a variety of molecular alterations. A relevant feature of aging is chronic lowgrade inflammation, termed "inflammaging." In type 2 diabetes mellitus (T2DM), many elements of aging appear earlier or are overrepresented, including consistent inflammaging. T2DM patients have an increased death rate, associated with an incremented inflammatory score. The source of this inflammation is debated. Recently, the senescence-associated secretory phenotype (SASP) has been proposed as the main origin of inflammaging in both aging and T2DM. Different pathogenic mechanisms linked to T2DM progression and complications development have been linked to senescence and SASP, that is, oxidative stress and endoplasmic reticulum (ER) stress. Here we review the latest data connecting oxidative and ER stress with the SASP in the context of aging and T2DM, with emphasis on endothelial cells (ECs) and endothelial dysfunction. Moreover, since current medical practice is insufficient to completely suppress the increased death rate of diabetic patients, we propose a SASP-centered view of T2DM as a futuristic therapeutic option, possibly opening new prospects by moving the attention from one-organ studies of diabetes complications to a wider targeting of the aging process.
\end{abstract}

\section{Introduction}

Aging is an intricate process that results from a combination of environmental, genetic, epigenetic, and stochastic factors [1]. A chronic proinflammatory status is a pervasive feature of aging. This chronic, low-grade, systemic inflammation occurring in the absence of overt infection (sterile inflammation) has been defined as "inflammaging" and represents a significant risk factor for morbidity and mortality in the elderly [2]. There is growing epidemiological evidence that a state of mild inflammation is associated with and predicts several age-related diseases (ARDs), including type 2 diabetes mellitus (T2DM) and its complications (e.g., cardiac death) [3-5]. According to the tenets of inflammaging, both the aging process per se and ARD development are fostered by an inescapable age-dependent proinflammatory drift [2]. The etiology of inflammaging and its contribution to ARDs development are the subject of intense research work.

The life expectancy of T2DM patients is about 6 years shorter than that of nondiabetic individuals of similar age [6]. Analysis of the factorial structure changes that take place during aging has disclosed that healthy aging involves a decrease in complexity and a concomitant increase in variability and inflammation. Interestingly, a decrease in complexity seems to arise earlier in diabetes patients [7], suggesting that under some respects diabetes may provide an interesting model of "accelerated aging." Diabetes patients may thus experience an accelerated aging process that increases the risk of developing 
frailty and morbidity and of an earlier death [8]. Although the role of inflammation in the pathogenesis of T2DM and its complications is well established $[5,9]$, the underlying molecular mechanisms are debated. Together with immunological factors, cellular senescence and the senescence-associated secretory phenotype (SASP) are currently held to be the largest contributors to inflammaging [1]; however, a key role of senescence in patients with the most common ARDs (e.g., diabetes) has yet to be conclusively demonstrated. Significantly, at least two major molecular changes responsible for diabetes complications and also associated with physiological aging and T2DM, that is, oxidative stress and endoplasmic reticulum (ER) stress $[10,11]$, have recently been related to senescence acquisition and/or SASP modulation [12, 13]. These findings suggest that the SASP can contribute to the endothelial dysfunction characterizing aging as well as T2DM.

Here we review the latest data connecting oxidative and ER stress with the SASP in the context of aging and T2DM, with emphasis on endothelial cells (ECs) and endothelial dysfunction. Moreover, since current lifestyle interventions and medications are unable to reduce the mortality of diabetic patients from cardiovascular disease, we also outline a gerontological, SASP-centered view of the vascular complications of diabetes that could provide a broader range of therapeutic options.

\section{Endothelial Senescence: A Central Player in Aging and Diabetes Complications}

Aging is accompanied by a progressive endothelial dysfunction that affects both diabetes patients and healthy individuals [14]. This widely accepted notion suggests that vascular aging-where vessel walls undergo profound remodeling including lumen enlargement, intimal and medial thickening, and increased vascular stiffness combined with loss of endothelial barrier integrity and function-is a key factor in organismal aging and in the development of diabetes complications [15-17]. These changes have recently been related to the onset of a proinflammatory program in ECs and vessel wall cells [18]. Inflammation is a nonspecific stereotypical response elicited by a variety of stimuli, many of which have been implicated in aging and T2DM $[1,2]$. We have designated the secretory phenotype acquired by ECs "endo-SASP" [19]. The rate of senescent cell accumulation in the body depends on the balance between pro- and antisenescence stimuli [20]. However, the sheer number (about 60 trillion) of ECs and the area covered by them (about 4000$7000 \mathrm{~m}^{2}$ ) suggest that SASP acquisition by these cells must exert a strong effect at the systemic level [21]. In particular, the buildup of senescent ECs is held to foster inflammaging. Indeed, ex vivo endothelium from relatively healthy aged and T2DM individuals shows chronic nuclear factor $-\kappa \mathrm{B}$ (NF- $\kappa \mathrm{B})$ expression and activation [22,23]. Moreover, blockade of NF$\kappa \mathrm{B}$-mediated inflammatory responses in the endothelium prolongs lifespan by preventing insulin resistance [24]. These findings clearly point at the endothelium as a key organ in the pathophysiology of aging and in diabetes complications.

\section{Senescence, SASP, and Inflammaging}

Cellular senescence is associated with the acquisition of the SASP, which is characterized by the activation of a proinflammatory transcriptional program and is held to drive ARD development through chronic secretion of a variety of factors $[1,25,26]$. Interestingly, the main pathways involved in cellular senescence regulation, such as the NF$\kappa \mathrm{B}$, the mechanistic target of rapamycin (mTOR), and the interleukin-1/NLR family pyrin domain containing 3 (IL1/NLRP3) inflammasome pathways, are master modulators of the aging rate [27-29]. In animal models, the number of senescent cells in specific organs predicts lifespan [30]. Notably, in animal models removal of senescent cells is sufficient to prolong lifespan and healthspan, as described in mice models of accelerated aging (INK-ATTAC mice) and in models of physiological aging [31,32]. Evidence that the number of senescent dermal fibroblasts correlates with ARD incidence has also been provided in humans [33].

A variety of exogenous and endogenous stimuli, including replicative exhaustion, oncogene activation, oncosuppressor loss, DNA damage, irradiation, viral infection, oxidative stress, and hyperglycemia, are capable of inducing a state of permanent cell cycle arrest accompanied by a sustained proinflammatory program. In proliferating human cells, progressive telomere erosion eventually leads to exposure of an uncapped free double-stranded chromosome end, triggering a permanent DNA damage response (DDR). The damage sensor ataxia telangiectasia mutated (ATM) is subsequently recruited to the uncapped telomeres, fostering the stabilization of tumor suppressor protein 53 (p53) and upregulating its target $\mathrm{p} 21$. In turn, p21 prevents inactivation of retinoblastoma tumor suppressor protein (RB), thus inhibiting entry into the $S$ phase of the cell cycle $[26,34]$. Other DNA-damaging stressors, such as ultraviolet radiation, chemotherapeutic drugs, hyperglycemia, and oncogenic Ras overexpression, also engage the ATM-p53-p21 axis [35-37]. This senescence pathway can also be engaged by loss of the tumor suppressor phosphatase and tensin homolog (PTEN) and overexpression of the S-phase E2F transcription factor 3 $(\mathrm{E} 2 \mathrm{~F} 3)[26,34] \cdot \mathrm{p} 16^{\text {Ink4a }}$ is a further barrier to proliferation, preventing cyclin-dependent kinase-4/6- (CDK4-/CDK6-) mediated inactivation of $\mathrm{RB}$, thus blocking cell cycle progression [26, 34]. This mechanism can act either alone or in combination with the p53-p21 pathway, depending on stressor or cell type. Therefore, different prosenescence stimuli may engage different pathways $[26,34]$, leading to partially different gene expression patterns [38]. However, a large panel of secreted factors is characteristic of the senescence state induced by all stimuli in all cell types. Indeed, the SASP involves release of hundreds of molecules [35-38], of which interleukin- (IL-) $1 \alpha / \beta$, IL-6, IL-8, transforming growth factor- (TGF-) $\beta$, and tumor necrosis factor- (TNF-) $\alpha$ are the most common and best characterized $[35,36$, 38]. Some of these cytokines can induce or reinforce the senescent phenotype by acting in an autocrine and paracrine manner, spreading senescence via a "bystander effect" [36]. In particular, IL- $1 \alpha$ appears to be the main upstream regulator of the SASP, while IL- $1 \beta$ and TGF- $\beta$ are mediators of senescence 
transmission, and IL- 6 and IL- 8 reinforce autocrine senescence $[36,39-42]$. These data suggest that regardless of the "first culprit," tissue ages "senescence by senescence."

A number of adaptor proteins have been shown to control SASP factor secretion. The SASP is mostly induced by $N F-\kappa B$, the main immunological transcription factor [43]. Upstream, p38 [44], JAK [45], and other MAP kinases [46], are all involved in SASP induction and control. Interestingly, Jak2/Stat3 pathway inhibition redesigns the SASP, suppressing the secretion of certain factors and increasing others (i.e., MCP-1), indicating that selective SASP modulation could be feasible [47]. Finally, highly interesting results in terms of SASP suppression have been obtained with the mTOR inhibitor rapamycin $[48,49]$, which has long been known to extend lifespan and healthspan in mice [50]. mTOR controls SASP protein secretion by enhancing IL- $1 \alpha$ and MAP kinaseactivated protein kinase 2 (MAPKAP2) translation [48, 49].

In vivo measurement of senescence and the SASP is of critical importance. Number of senescence-associated heterochromatin foci (SAHF), number and phosphorylation of $\gamma$ $\mathrm{H} 2 \mathrm{AX}$ foci, levels of the heterochromatin protein HP1 $\gamma$, P53binding protein 1 (53BP1), H3K9me3, and other markers have been measured both in senescent cells and nonsenescent cells "stressed" in various ways (e.g., radiation, hyperglycemia, oxidative stress, and ER stress) [51], suggesting a close interconnection among such phenomena. The expression of p16Ink4a, the end effector of cell cycle arrest, and SA- $\beta$ galactosidase enzyme activity are currently the most widely used surrogate markers of senescence [26], even though the possibility of false positive results has been reported for both [52, 53]. Similarly, the inflammatory components of the SASP are shared by nearly all types of inflammatory responses. Unfortunately, research efforts are still hampered by the lack of a universally accepted standard to identify senescent cells and determine their functional significance in vivo [26]. In vivo senescence probably encompasses a spectrum of states ranging from low to high SASP expression, depending on its inducers (replication, oxidative stress, oncogene activation) and cell types, among other factors. The chronic senescent state characteristic of natural aging is likely to be a complex phenomenon induced by some combination of telomere attrition, (oxidative) DNA damage, ER stress, and other slowly accumulating forms of macromolecular damage [26].

Interventions directed at preventing the adverse effects associated with the SASP are being explored $[25,26]$. The most promising strategies involve delaying cellular senescence [30]; SASP switch-off [48, 49]; SASP factor modulation $[34,47]$; and selective removal or killing of existing senescent cells (senolytics or senotherapy) [25, 54]. Selective targeting and killing of senescent cells without damaging neighboring, healthy cells requires identifying senescence-associated markers and devising strategies to exploit them $[25,26]$. High-throughput "omics" technologies (i.e., genomics, metabolomics, metagenomics, and transcriptomics) are being applied to discover such markers [55]. Highly promising results are coming from work on SASP suppressor and senolytic agents [26, 48-54].

\section{SASP, Inflammaging, and Type 2 Diabetes}

Several ARDs, including T2DM and cardiovascular diseases, share a chronic, low-grade inflammatory state $[1,2]$. All lines of evidence point at low-grade inflammation as a key T2DM therapeutic target [9]. According to a recent hypothesis, the buildup of cells expressing the SASP may promote the development of both diabetes and its vascular complications $[26,56]$. SASP genes (i.e., IL- $1 \alpha$, IL- $1 \beta$, IL-6, and TNF- $\alpha$ ) are chronically activated in cells and tissues from diabetes patients [23]. The role of senescence in the development of T2DM and related vascular complications and whether it predates or follows the onset of low-grade inflammation and vascular complications are the subject of intense research work. An outstanding question is whether the glucose-related metabolic perturbations of T2DM promote telomere attrition and increase DNA oxidative damage or accelerated cellular senescence in multiple cell types, including $\beta$ cells, adipose tissue, and endothelium, is a pathogenic mechanism involved in T2DM development and progression [56]. Epigenetic modifications leading to chronic inflammation have been described in ECs and immune cells of diabetes patients also in the absence of replicative senescence biomarkers $[23,57$, 58]. However, the majority of the inflammatory mediators involved in the vascular complications of diabetes, which are induced in vitro in ECs and immune cells by hyperglycemia, are the SASP-modulating and SASP-released molecules, like NF- $\kappa$ B, IL-1, IL- 6 , TNF- $\alpha$, and vascular cell adhesion molecule-1 (VCAM-1) $[23,58]$, suggesting that the SASP has a causal role in maintaining the chronic, systemic inflammation that is associated with diabetes. A comparative analysis of gene expression in replicative and hyperglycemia-induced senescence could shed some light on the question. Moreover, circulating cytokine concentrations can be increased by hyperglycemia, also in an acute manner, through an oxidative mechanism, whose effect is more marked in subjects with impaired glucose tolerance [59]. High-glycemic index carbohydrate increases NF- $\kappa \mathrm{B}$ activation in peripheral blood mononuclear cells (PBMCs) and this proinflammatory effect could be partially reversed by prandial basal insulin treatment $[60,61]$. Hyperglycemia thus promotes the acquisition of a proinflammatory cellular phenotype that may be defined as a diabetes- or hyperglycemia-associated secretory phenotype (DASP or HASP). The high-level oxidative stress induced by hyperglycemia and other imbalances associated with T2DM (i.e., altered hormonal status and lipid metabolism, epigenetic alterations, and low-grade inflammation) have the potential to foster premature senescence $[56,60]$. Senescent cell accumulation can therefore contribute to spread senescence at the systemic level, further fueling low-grade inflammation and promoting T2DM progression $[56,57]$. Consequently, senescent cells might be part of a pathogenic loop both as a cause and a consequence of metabolic changes and tissue damage in diabetes [56, 62]. This hypothesis is partially supported by the observation that leukocyte telomere length (LTL), a surrogate marker of senescence, has a nonlinear association with incident diabetes, indicating that it could serve to predict T2DM development [63]. Moreover, a clear, inverse age-dependent association between LTL and insulin 
resistance has been documented [64]. LTL is significantly shorter in T2DM patients compared with controls over a wide age range and is significantly associated with the presence and number of diabetes complications [65]. Finally, a strong association has been highlighted between telomere length and the presence of some diabetes complications and general arterial aging $[66,67]$.

Evidence of significant in situ senescence of cells with a functional SASP in adipose tissue (mainly ECs and adipocyte progenitors) from obese subjects has been linked to T2DM development $[37,68]$. As regards pancreatic $\beta$-cells, even though aging in itself does not appear to be critical in inducing tissue dysfunction [69, 70], increased $\beta$-cell senescence during aging and a limited regenerative potential have been described in senescent mouse pancreas [71]. After the initial in vitro demonstration that exposure to high glucose levels induces EC senescence [72], in vivo evidence of increased senescence has been provided in atherosclerotic plaques and kidney tissue of diabetes patients, suggesting the relevance of this phenomenon to T2DM vascular complications [73, 74]. Moreover, mounting evidence suggests an important role for the inflammasome platform in both T2DM and atherosclerosis [75, 76]. The NOD-like receptor- (NLR-) caspase 1-IL-1 $\beta$ cascade can be activated by endogenous metabolism or injury-derived byproducts called damageassociated pattern molecules (DAMPs), resulting in chronic secretion of inflammatory cytokines [77]. Strikingly, the inflammasome controls transmission of the SASP senescence signal [39]. In turn, it is widely accepted that oxidative stress and macromolecular damage play a major role in controlling the inflammasome platform [75, 78, 79], mainly through the Trx/TXNIP complex, linking redox status, ER stress, and inflammation. NLRs likely respond to some generic cellular stress signals induced by the multiple molecules that trigger its activation. In addition to NLR activation, tolllike receptor (TLR) activation has also been proposed to be closely involved in T2DM and its complications, supporting a role for innate immunity and probably microbiota in the diabetic inflammatory milieu $[80,81]$.

Clinical trials involving IL-1 blockade in T2DM patients have provided promising results. Anakinra (a recombinant human IL-1 receptor antagonist) improved glycemia and $\beta$ cell secretory function and reduced the levels of systemic inflammation markers [82]. Canakinumab, a human monoclonal antibody that neutralizes IL- $1 \beta$, also significantly reduced inflammation and glycated hemoglobin in diabetes patients $[83,84]$. Large clinical trials are exploring the potential of IL-1 antagonism to prevent cardiovascular and other diabetes complications [85]. Anticytokine agents are commonly used to treat autoimmune diseases, which are characterized by chronic inflammation, endothelial dysfunction, accelerated aging, and a heavy burden of senescent cells $[86,87]$. The three licensed anti-TNF- $\alpha$ biologics have shown effectiveness in improving endothelial function; similar results have been obtained with anti-IL-6 treatment [88]. However, clinical trials that block these cytokines in T2DM patients are yet to be set up.

The pathophysiology of T2DM complications is characterized by increased oxidative stress, nonenzymatic glycation, and PKC overactivation as the main molecular changes involving micro- and macrovascular compartments [89]. However, targeting these changes with innovative therapies has had limited success in slowing down disease progression and the development of complications [90], indicating that not all the imbalances experienced by diabetes patients can be addressed by one-target treatments. The pathways involved in the vascular complications of T2DM are complex, interlinked, and self-perpetuating. It is unlikely for a single druggable pathway to prevent their onset, probably also because the intricate connections among the mechanisms giving rise to diabetes complications create redundancy [90]. Moreover, current diabetes treatments are unable to halt the development of vascular complications, especially in patients with long-standing disease [91]. Therefore, if a large role for senescence and the SASP in T2DM is confirmed, new therapies aimed to remove senescent cells or counter the noxious effect of the SASP might be suitable alternatives to slow down T2DM progression and delay complication onset. For instance, rapamycin, the main SASP-suppressing agent, reduces renal hypertrophy in diabetic mice and slows the progression of diabetic kidney disease in rats, even without reducing blood glucose [92]. Prevention of age-related macular degeneration-like retinopathy by rapamycin has also been reported [93]. A theoretical model where mTOR is viewed as the central mediator of insulin resistance and diabetes complications has been proposed [94]. A putative antisenescence in vivo role [95] and an anti-SASP in vitro action [96] have been proposed for metformin, currently the most effective antidiabetes agent in terms of prevention of its vascular complications [91]. Finally, an agent interfering with the glycolysis pathway (2-deoxyglucose) has been shown to selectively affect senescent cells by exerting an antisurvival action, thus attenuating inflammation [97].

\section{Oxidative Stress-Induced Senescence and Redox Control of the SASP}

A large number of studies have documented the ability of antioxidant compounds to delay senescence onset in vitro. In ECs this has clearly been demonstrated for coenzyme Q10 [98], $\mathrm{N}$-acetyl cysteine [99], and a stable vitamin $\mathrm{C}$ analog [100]. However, clinical studies have failed to confirm the benefit of antioxidants and vitamins in cardiovascular disease prevention and management [101]. A number of reasons may account for this: (i) nonoptimal pharmacokinetic profiles, preventing antioxidants from reaching the endothelium or from maintaining a stable concentration of the agent over time; (ii) overestimation of the in vitro effect of antioxidants, since experiments are usually performed under normal oxygen tension, whereas oxygen levels in the body are considerably lower, especially in deep tissue layers; (iii) possible downregulation of cellular antioxidant defenses (i.e., superoxide dismutase, catalase) following prolonged exposure to the antioxidant (pharmacodynamic tolerance); and (iv) the possible sensitivity of the endothelium, which is continuously exposed to various stressors, to reactive oxygen species (ROS) dosage and the existence of a putative beneficial effect of 
low ROS levels in vivo (hormesis) [102, 103]. Notably, recent experimental evidence strongly suggests that low ROS levels extend lifespan in different model organisms [102]. Therefore, a more useful definition of oxidative stress may be "a state where oxidative force exceeds the antioxidant systems due to loss of the balance between them" [104]. More promising results have been obtained in T2DM patients and animal models. Endothelial progenitor cells (EPCs) from diabetic donors undergo oxidative stress-induced premature senescence [105]. EC senescence, with p53 and p16 upregulation in endothelium, also occurs in the arteries of rats with T2DM [106]. Importantly, these animals display evidence of vascular dysfunction, and antioxidant treatment prevents endothelial senescence, ameliorating endothelial dysfunction. Similarly, diabetic patients treated with antioxidant compounds show improved endothelial function and inflammatory profiles [107]. However, improvement of long-term outcomes in T2DM patients has not been detected [108]. Enhancing cellular antioxidant defenses has been proposed as an alternative to antioxidant administration to restore EC physiological redox status and prevent T2DM- or aging-induced cardiovascular disease by reducing hyperglycemia- and/or aging-related damage [108]. For example, caloric restriction (CR), which has well-established lifespan-promoting action, exerts persistent antioxidant and anti-inflammatory cellular effects, preserving a youthful phenotype in several cell types including ECs. Among nutrient-sensitive factors, sirtuins, a family of $\mathrm{NAD}^{+}$-dependent deacetylases with epigenetic modulation activity, can prevent vascular endothelial replicative senescence by increasing antioxidant defense $[109,110]$. CR mimetics like resveratrol and other synthetic sirtuin activators confer broad health benefits including preservation of endothelial function and attenuation of low-grade inflammation, especially in obesity and T2DM models [109-111].

Besides senescence, the SASP is also controlled by the cellular redox status [12]. IL-1 $\alpha$ is a key senescence-associated proinflammatory cytokine acting as a critical upstream regulator of the SASP. Senescence-associated shifts in steadystate $\mathrm{H}_{2} \mathrm{O}_{2}$ and intracellular $\mathrm{Ca}^{2+}$ levels induce increased IL$1 \alpha$ expression and processing. Increased intracellular $\mathrm{Ca}^{2+}$ promotes calpain activation and the proteolytic cleavage of IL-1 $\alpha$ [12]. Antioxidants and low oxygen tension prevented senescence-associated IL- $1 \alpha$ expression and reduced the expression of SASP components IL-6 and IL-8. Similarly, $\mathrm{Ca}^{2+}$ chelation or calpain inhibition impaired senescenceassociated processing of IL-1 $\alpha$ and its ability to induce downstream cytokine expression [12].

\section{Endoplasmic Reticulum Stress, Diabetes, and the SASP}

Over the past decade, ER stress has emerged as a new factor in the pathogenesis of diabetes and its complications. A considerable number of recent studies have highlighted its role in the onset of insulin resistance, hyperglycemia, and endothelial dysfunction [112].

Pathological conditions involving altered ER homeostasis, such as overexpression of misfolded proteins, low ER chaperone levels and $\mathrm{Ca}^{2+}$ content, ER phospholipid depletion and cholesterol accumulation, and changes in the redox status that occur in diabetes, induce a state of ER stress that leads to activation of Unfolded Protein Response (UPR) [112, 113]. ER stress is an imbalance between the proper folding and the secretory capacity of the ER that results in accumulation of misfolded proteins. Loss of ER homeostasis activates the ER stress response, a crucial adaptive mechanism in secretory cells, which serves to dynamically expand ER size and capacity to meet the functional demand placed on the exocytosis pathway [112]. UPR markers are overexpressed in the liver and adipose tissue of diabetic rodents [113] as well as in ex vivo samples from T2DM patients [114].

Chronic ER stress and UPR activation may also result in ROS buildup, which induces a state of oxidative stress [115]. ROS overproduction and the development of oxidative stress can thus be viewed both as a cause and a consequence of ER stress. ROS accumulation is due to UPRstimulated upregulation of chaperone proteins involved in disulfide bond formation in the ER lumen. Chaperones use oxidation/reduction reactions, with molecular oxygen as the final electron recipient. The reduced molecular oxygen accumulates during increased protein folding due to UPR activation, resulting in cell toxicity [116].

Diabetes or hyperglycemia induce ER stress in many organs [112-116], including endothelium [117]. Many biochemical and molecular imbalances found in T2DM subjects are ER stress inducers. Altered nutrient availability, free fatty acids, cytokines, perturbations in calcium transients, oxidative stress, and hypoxia are all capable of triggering accumulation of unfolded ER proteins [118].

Interestingly, recent papers strongly link ER stress with SASP. In particular, a negative feedback loop mediated by macro H2A1.1 limits ER stress during senescence by suppressing SASP gene expression [13]. ER stress is a feature of senescence triggered by the expression of some secreted SASP molecules $[119,120]$. The formation of correct disulfide bonds is critical for many SASP components. For instance, CXC-type cytokines such as IL-8 require the formation of two disulfide bonds for folding and function [121]. Disulfide bond rearrangement in the ER is catalyzed by protein disulfide isomerases (PDIs), which are critical for the folding of disulfidecontaining secreted factors [122]. Increased PDI activity due to unfolded proteins can then increase ROS levels, chiefly through NADPH oxidase 4 (NOX4) upregulation [122]. In turn, NOX4 is a prooxidant enzyme capable of triggering senescence [123].

Canonical UPR pathways try to mitigate ER stress through several mechanisms that include increased ER chaperone synthesis, inhibition of translation, and regulated IRE1dependent decay (RIDD) of mRNA [124]. The increased ROS levels caused by ER stress induce DNA damage, activating a DDR involving ATM [125]. Active ATM elicits macro H2A1 removal from SASP genes, keeping ER stress in check, a process called reactive oxygen and ATM-mediated macro H2A1 mobilization (ROAMM) pathway [13]. The ROAMM pathway explains why ATM inhibition leads to steep increases in SASP gene expression and ER stress. In the absence of ATM-mediated negative feedback from ER stress, the SASP 
positive feedback loop runs unopposed, inducing a dramatic increase in both SASP expression and ER stress [13].

\section{Conclusions and Future Directions}

Epidemiological, biochemical, and molecular lines of evidence suggest that aging and T2DM share a number of important features that include oxidative stress, ER stress, endothelial dysfunction, and low-grade inflammation. SASP is modulated by or modulates all these mechanisms and may be a druggable element of connection. Several lines of research are trying to harness some molecular features of aging in the context of T2DM, like sirtuin family activators, mTOR inhibitors, and ER stress modulators. The route to clinical translation requires further, decisive insights into the role of senescence/SASP in T2DM. For instance, crossing T2DM mice models with the INK-ATTAC model (characterized by automated clearance of senescent cells) would provide definitive information. T2DM could be the ideal model to test senolytics and other SASP-modifying drugs. T2DM is very likely to benefit from a shift from single pathway blockade to broader targeting of the senescence process. A SASPcentered or simply gerontological view of T2DM could open new prospects by moving the focus from single organ studies of diabetes complications to their long-term consequences, possibly improving the condition of those patients whose higher risk of death is not mitigated by current disease management approaches.

\section{Competing Interests}

None of the authors have competing interests.

\section{Authors' Contributions}

Francesco Prattichizzo, Valeria De Nigris, Fabiola Olivieri, and Antonio Ceriello conceived the idea and have been involved in paper conception and drafting; Lucia La Sala and Antonio Domenico Procopio revised the paper critically. All authors have given their final approval of this version to be published. All authors have read and approved the final paper. Francesco Prattichizzo and Valeria De Nigris equally contributed to the work.

\section{Acknowledgments}

The authors are grateful to Word Designs for the language revision (http://www.silviamodena.com/). Francesco Prattichizzo is the holder of a postdoctoral fellowship from Università Politecnica delle Marche to be exploited abroad.

\section{References}

[1] C. Franceschi and J. Campisi, "Chronic inflammation (inflammaging) and its potential contribution to age-associated diseases," Journals of Gerontology, Series A: Biological Sciences and Medical Sciences, vol. 69, supplement 1, pp. S4-S9, 2014.
[2] C. Franceschi, M. Bonafè, S. Valensin et al., "Inflamm-aging. An evolutionary perspective on immunosenescence," Annals of the New York Academy of Sciences, vol. 908, pp. 244-254, 2000.

[3] The Interleukin-6 Receptor Mendelian Randomisation Analysis (IL6R MR) Consortium, "The interleukin-6 receptor as a target for prevention of coronary heart disease: a mendelian randomisation analysis," The Lancet, vol. 379, no. 9822, pp. 12141224, 2012.

[4] M. Maggio, J. M. Guralnik, D. L. Longo, and L. Ferrucci, "Interleukin-6 in aging and chronic disease: a magnificent pathway," Journals of Gerontology Series A: Biological Sciences and Medical Sciences, vol. 61, no. 6, pp. 575-584, 2006.

[5] X. Wang, W. Bao, J. Liu et al., "Inflammatory markers and risk of type 2 diabetes: a systematic review and meta-analysis," Diabetes Care, vol. 36, no. 1, pp. 166-175, 2013.

[6] E. S. Huang, N. Laiteerapong, J. Y. Liu, P. M. John, H. H. Moffet, and A. J. Karter, "Rates of complications and mortality in older patients with diabetes mellitus: the diabetes and aging study," JAMA Internal Medicine, vol. 174, no. 2, pp. 251-258, 2014.

[7] L. Spazzafumo, F. Olivieri, A. M. Abbatecola et al., "Remodelling of biological parameters during human ageing: evidence for complex regulation in longevity and in type 2 diabetes," Age, vol. 35, no. 2, pp. 419-429, 2013.

[8] S. Perkisas and M. Vandewoude, "Where frailty meets diabetes," Diabetes/Metabolism Research and Reviews, vol. 32, supplement 1, pp. 261-267, 2016.

[9] M. Y. Donath, "Targeting inflammation in the treatment of type 2 diabetes: time to start," Nature Reviews Drug Discovery, vol.13, no. 6, pp. 465-476, 2014.

[10] F. Bonomini, L. F. Rodella, and R. Rezzani, "Metabolic syndrome, aging and involvement of oxidative stress," Aging and Disease, vol. 6, no. 2, pp. 109-120, 2015.

[11] M. Muriach, M. Flores-Bellver, F. J. Romero, and J. M. Barcia, "Diabetes and the brain: oxidative stress, inflammation, and autophagy," Oxidative Medicine and Cellular Longevity, vol. 2014, Article ID 102158, 9 pages, 2014.

[12] D. A. McCarthy, R. R. Clark, T. R. Bartling, M. Trebak, and J. A. Melendez, "Redox control of the senescence regulator interleukin- $1 \alpha$ and the secretory phenotype," The Journal of Biological Chemistry, vol. 288, no. 45, pp. 32149-32159, 2013.

[13] H. Chen, P. D. Ruiz, W. M. McKimpson, L. Novikov, R. N. Kitsis, and M. J. Gamble, "MacroH2A1 and ATM play opposing roles in paracrine senescence and the senescence-associated secretory phenotype," Molecular Cell, vol. 59, no. 5, pp. 719-731, 2015.

[14] M. D. Herrera, C. Mingorance, R. Rodríguez-Rodríguez, and M. Alvarez de Sotomayor, "Endothelial dysfunction and aging: an update," Ageing Research Reviews, vol. 9, no. 2, pp. 142-152, 2010.

[15] L. Rodríguez-Mañas, M. El-Assar, S. Vallejo et al., "Endothelial dysfunction in aged humans is related with oxidative stress and vascular inflammation," Aging Cell, vol. 8, no. 3, pp. 226-238, 2009.

[16] R. P. Brandes, I. Fleming, and R. Busse, "Endothelial aging," Cardiovascular Research, vol. 66, no. 2, pp. 286-294, 2005.

[17] G. Favero, C. Paganelli, B. Buffoli, L. F. Rodella, and R. Rezzani, "Endothelium and its alterations in cardiovascular diseases: life style intervention," BioMed Research International, vol. 2014, Article ID 801896, 28 pages, 2014.

[18] M. Wang, R. E. Monticone, and E. G. Lakatta, "Proinflammation of aging central arteries: a mini-review," Gerontology, vol. 60, no. 6, pp. 519-529, 2014. 
[19] F. Prattichizzo, M. Bonafè, A. Ceka et al., "Endothelial cell senescence and inflammaging: MicroRNAs as biomarkers and innovative therapeutic tools," Current Drug Targets, vol. 17, no. 4, pp. 388-397, 2016.

[20] C. López-Otín, M. A. Blasco, L. Partridge, M. Serrano, and G. Kroemer, "The hallmarks of aging," Cell, vol. 153, no. 6, pp. X1194-X1217, 2013.

[21] W. C. Aird, "Endothelial cell heterogeneity and atherosclerosis," Current Atherosclerosis Reports, vol. 8, no. 1, pp. 69-75, 2006.

[22] A. J. Donato, A. D. Black, K. L. Jablonski, L. B. Gano, and D. R. Seals, "Aging is associated with greater nuclear NFאB, reduced $\mathrm{I} \kappa \mathrm{B} \alpha$, and increased expression of proinflammatory cytokines in vascular endothelial cells of healthy humans," Aging Cell, vol. 7, no. 6, pp. 805-812, 2008.

[23] M. E. Cooper and A. El-Osta, "Epigenetics: mechanisms and implications for diabetic complications," Circulation Research, vol. 107, no. 12, pp. 1403-1413, 2010.

[24] Y. Hasegawa, T. Saito, T. Ogihara et al., "Blockade of the nuclear factor- $\kappa \mathrm{B}$ pathway in the endothelium prevents insulin resistance and prolongs life spans," Circulation, vol. 125, no. 9, pp. 1122-1133, 2012.

[25] T. Tchkonia, Y. Zhu, J. Van Deursen, J. Campisi, and J. L. Kirkland, "Cellular senescence and the senescent secretory phenotype: therapeutic opportunities," Journal of Clinical Investigation, vol. 123, no. 3, pp. 966-972, 2013.

[26] B. G. Childs, M. Durik, D. J. Baker, and J. M. van Deursen, "Cellular senescence in aging and age-related disease: from mechanisms to therapy," Nature Medicine, vol. 21, no. 12, pp. 1424-1435, 2015.

[27] G. Zhang, J. Li, S. Purkayastha et al., "Hypothalamic programming of systemic ageing involving IKK- $\beta$, NF- $\kappa$ B and GnRH," Nature, vol. 497, no. 7448, pp. 211-216, 2013.

[28] S. C. Johnson, P. S. Rabinovitch, and M. Kaeberlein, "mTOR is a key modulator of ageing and age-related disease," Nature, vol. 493, no. 7432, pp. 338-345, 2013.

[29] Y.-H. Youm, R. W. Grant, L. R. McCabe et al., "Canonical Nlrp3 inflammasome links systemic low-grade inflammation to functional decline in aging," Cell Metabolism, vol. 18, no. 4, pp. 519-532, 2013.

[30] D. Jurk, C. Wilson, J. F. Passos et al., "Chronic inflammation induces telomere dysfunction and accelerates ageing in mice," Nature Communications, vol. 2, article 4172, 2014.

[31] D. J. Baker, T. Wijshake, T. Tchkonia et al., "Clearance of p16 ${ }^{\text {Ink4a }}$-positive senescent cells delays ageing-associated disorders," Nature, vol. 479, no. 7372, pp. 232-236, 2011.

[32] D. J. Baker, B. G. Childs, M. Durik et al., "Naturally occurring p16 ${ }^{\text {Ink4a }}$-positive cells shorten healthy lifespan," Nature, vol. 530, no. 7589, pp. 184-189, 2016.

[33] M. E. C. Waaijer, W. E. Parish, B. H. Strongitharm et al., "The number of p16INK4a positive cells in human skin reflects biological age," Aging Cell, vol. 11, no. 4, pp. 722-725, 2012.

[34] D. Di Mitri and A. Alimonti, "Non-cell-autonomous regulation of cellular senescence in cancer," Trends in Cell Biology, vol. 26, no. 3, pp. 215-226, 2015.

[35] J. Campisi and F. d'Adda di Fagagna, "Cellular senescence: when bad things happen to good cells," Nature Reviews Molecular Cell Biology, vol. 8, no. 9, pp. 729-740, 2007.

[36] A. Freund, A. V. Orjalo, P.-Y. Desprez, and J. Campisi, "Inflammatory networks during cellular senescence: causes and consequences," Trends in Molecular Medicine, vol. 16, no. 5, pp. 238246, 2010 .
[37] A. Villaret, J. Galitzky, P. Decaunes et al., "Adipose tissue endothelial cells from obese human subjects: differences among depots in angiogenic, metabolic, and inflammatory gene expression and cellular senescence," Diabetes, vol. 59, no. 11, pp. 2755-2763, 2010.

[38] M. Purcell, A. Kruger, and M. A. Tainsky, "Gene expression profiling of replicative and induced senescence," Cell Cycle, vol. 13, no. 24, pp. 3927-3937, 2014.

[39] J. C. Acosta, A. Banito, T. Wuestefeld et al., "A complex secretory program orchestrated by the inflammasome controls paracrine senescence," Nature Cell Biology, vol. 15, no. 8, pp. 978-990, 2013.

[40] A. V. Orjalo, D. Bhaumik, B. K. Gengler, G. K. Scott, and J. Campisi, "Cell surface-bound IL-1 $\alpha$ is an upstream regulator of the senescence-associated IL-6/IL-8 cytokine network," Proceedings of the National Academy of Sciences of the United States of America, vol. 106, no. 40, pp. 17031-17036, 2009.

[41] T. Kuilman, C. Michaloglou, L. C. W. Vredeveld et al., "Oncogene-induced senescence relayed by an interleukindependent inflammatory network," Cell, vol. 133, no. 6, pp.10191031, 2008.

[42] T. Kuilman, C. Michaloglou, W. J. Mooi, and D. S. Peeper, "The essence of senescence," Genes and Development, vol. 24, no. 22, pp. 2463-2479, 2010.

[43] A. Salminen, A. Kauppinen, and K. Kaarniranta, "Emerging role of NF- $\kappa \mathrm{B}$ signaling in the induction of senescence-associated secretory phenotype (SASP)," Cellular Signalling, vol. 24, no. 4, pp. 835-845, 2012.

[44] A. Freund, C. K. Patil, and J. Campisi, "P38MAPK is a novel DNA damage response-independent regulator of the senescence-associated secretory phenotype," The EMBO Journal, vol. 30, no. 8, pp. 1536-1548, 2011.

[45] M. Xu, T. Tchkonia, H. Ding et al., "JAK inhibition alleviates the cellular senescence-associated secretory phenotype and frailty in old age," Proceedings of the National Academy of Sciences, vol. 112, no. 46, pp. E6301-E6310, 2015.

[46] M. Ferrand, O. Kirsh, A. Griveau et al., "Screening of a kinase library reveals novel pro-senescence kinases and their common NF- $\kappa$ B-dependent transcriptional program," Aging, vol. 7, no. 11, pp. 986-1003, 2015.

[47] A. Toso, A. Revandkar, D. Di Mitri et al., "Enhancing chemotherapy efficacy in pten-deficient prostate tumors by activating the senescence-associated antitumor immunity," Cell Reports, vol. 9, no. 1, pp. 75-89, 2014.

[48] R.-M. Laberge, Y. Sun, A. V. Orjalo et al., "MTOR regulates the pro-tumorigenic senescence-associated secretory phenotype by promoting IL1A translation," Nature Cell Biology, vol. 17, no. 8, pp. 1049-1061, 2015.

[49] N. Herranz, S. Gallage, M. Mellone et al., "mTOR regulates MAPKAPK2 translation to control the senescence-associated secretory phenotype," Nature Cell Biology, vol. 17, no. 9, pp. 1205-1217, 2015.

[50] D. E. Harrison, R. Strong, Z. D. Sharp et al., "Rapamycin fed late in life extends lifespan in genetically heterogeneous mice," Nature, vol. 460, no. 7253, pp. 392-395, 2009.

[51] T. Sperka, J. Wang, and K. L. Rudolph, "DNA damage checkpoints in stem cells, ageing and cancer," Nature Reviews Molecular Cell Biology, vol. 13, no. 9, pp. 579-590, 2012.

[52] A. N. Akbar and S. M. Henson, "Are senescence and exhaustion intertwined or unrelated processes that compromise immunity?" Nature Reviews Immunology, vol. 11, no. 4, pp. 289-295, 2011. 
[53] I. Bursuker, J. M. Rhodes, and R. Goldman, " $\alpha$-Galactosidasean indicator of the maturational stage of mouse and human mononuclear phagocytes," Journal of Cellular Physiology, vol. 112, no. 3, pp. 385-390, 1982.

[54] J. Chang, Y. Wang, L. Shao et al., "Clearance of senescent cells by ABT263 rejuvenates aged hematopoietic stem cells in mice," Nature Medicine, vol. 22, no. 1, pp. 78-83, 2016.

[55] A. M. Valdes, D. Glass, and T. D. Spector, "Omics technologies and the study of human ageing," Nature Reviews Genetics, vol. 14, no. 9, pp. 601-607, 2013.

[56] A. K. Palmer, T. Tchkonia, N. K. LeBrasseur, E. N. Chini, M. $\mathrm{Xu}$, and J. L. Kirkland, "Cellular senescence in type 2 diabetes: a therapeutic opportunity," Diabetes, vol. 64, no. 7, pp. 2289-2298, 2015.

[57] F. Olivieri, L. Spazzafumo, M. Bonafè et al., "MiR-21-5p and miR-126a-3p levels in plasma and circulating angiogenic cells: relationship with type 2 diabetes complications," Oncotarget, vol. 6, no. 34, pp. 35372-35382, 2015.

[58] F. Prattichizzo, A. Giuliani, A. Ceka et al., "Epigenetic mechanisms of endothelial dysfunction in type 2 diabetes," Clinical Epigenetics, vol. 7, no. 1, article 56, 2015.

[59] K. Esposito, F. Nappo, R. Marfella et al., "Inflammatory cytokine concentrations are acutely increased by hyperglycemia in humans: role of oxidative stress," Circulation, vol. 106, no. 16, pp. 2067-2072, 2002.

[60] P. J. Beisswenger, W. V. Brown, A. Ceriello et al., "Mealinduced increases in C-reactive protein, interleukin-6 and tumour necrosis factor $\alpha$ are attenuated by prandial + basal insulin in patients with Type 2 diabetes," Diabetic Medicine, vol. 28, no. 9, pp. 1088-1095, 2011.

[61] S. Dickinson, D. P. Hancock, P. Petocz, A. Ceriello, and J. BrandMiller, "High-glycemic index carbohydrate increases nuclear factor- $\kappa \mathrm{B}$ activation in mononuclear cells of young, lean healthy subjects," American Journal of Clinical Nutrition, vol. 87, no. 5, pp. 1188-1193, 2008.

[62] R. Testa and A. Ceriello, "Pathogenetic loop between diabetes and cell senescence," Diabetes Care, vol. 30, no. 11, pp. 29742975, 2007.

[63] J. Zhao, Y. Zhu, J. Lin et al., "Short leukocyte telomere length predicts risk of diabetes in American Indians: the strong heart family study," Diabetes, vol. 63, no. 1, pp. 354-362, 2014.

[64] I. Strazhesko, O. Tkacheva, S. Boytsov et al., "Association of insulin resistance, arterial stiffness and telomere length in adults free of cardiovascular diseases," PLoS ONE, vol.10, no. 8, Article ID e0136676, 2015.

[65] R. Testa, F. Olivieri, C. Sirolla et al., "Leukocyte telomere length is associated with complications of type 2 diabetes mellitus," Diabetic Medicine, vol. 28, no. 11, pp. 1388-1394, 2011.

[66] F. Olivieri, M. Lorenzi, R. Antonicelli et al., "Leukocyte telomere shortening in elderly Type2DM patients with previous myocardial infarction," Atherosclerosis, vol. 206, no. 2, pp. 588-593, 2009.

[67] E. N. Dudinskaya, O. N. Tkacheva, M. V. Shestakova et al., "Short telomere length is associated with arterial aging in patients with type 2 diabetes mellitus," Endocrine Connections, vol. 4, no. 3, pp. 136-143, 2015.

[68] T. Tchkonia, D. E. Morbeck, T. Von Zglinicki et al., "Fat tissue, aging, and cellular senescence," Aging Cell, vol. 9, no. 5, pp. 667684,2010

[69] D. Avrahami, C. Li, J. Zhang et al., "Aging-dependent demethylation of regulatory elements correlates with chromatin state and improved $\beta$ cell function," Cell Metabolism, vol. 22, no. 4, pp. 619-632, 2015.

[70] S. Chera, D. Baronnier, L. Ghila et al., "Diabetes recovery by age-dependent conversion of pancreatic $\delta$-cells into insulin producers," Nature, vol. 514, no. 7523, pp. 503-507, 2014.

[71] J. Krishnamurthy, M. R. Ramsey, K. L. Ligon et al., "p16INK4a induces an age-dependent decline in islet regenerative potential," Nature, vol. 443, no. 7110, pp. 453-457, 2006.

[72] T. Yokoi, K. Fukuo, O. Yasuda et al., "Apoptosis signal-regulating kinase 1 mediates cellular senescence induced by high glucose in endothelial cells," Diabetes, vol. 55, no. 6, pp. 1660-1665, 2006.

[73] T. Hayashi, H. Kotani, T. Yamaguchi et al., "Endothelial cellular senescence is inhibited by liver $\mathrm{X}$ receptor activation with an additional mechanism for its atheroprotection in diabetes," Proceedings of the National Academy of Sciences of the United States of America, vol. 111, no. 3, pp. 1168-1173, 2014.

[74] D. Verzola, M. T. Gandolfo, G. Gaetani et al., "Accelerated senescence in the kidneys of patients with type 2 diabetic nephropathy," American Journal of Physiology-Renal Physiology, vol. 295, no. 5, pp. F1563-F1573, 2008.

[75] K. Schroder, R. Zhou, and J. Tschopp, “The NLRP3 inflammasome: a sensor for metabolic danger?” Science, vol. 327, no. 5963, pp. 296-300, 2010.

[76] L. Bessueille and D. Magne, "Inflammation: a culprit for vascular calcification in atherosclerosis and diabetes," Cellular and Molecular Life Sciences, vol. 72, no. 13, pp. 2475-2489, 2015.

[77] J. J. Shin, E. K. Lee, T. J. Park, and W. Kim, "Damage-associated molecular patterns and their pathological relevance in diabetes mellitus," Ageing Research Reviews Part A, vol. 24, pp. 66-76, 2015.

[78] A. Abderrazak, T. Syrovets, D. Couchie et al., "NLRP3 inflammasome: from a danger signal sensor to a regulatory node of oxidative stress and inflammatory diseases," Redox Biology, vol. 4, pp. 296-307, 2015.

[79] J. Tschopp and K. Schroder, "NLRP3 inflammasome activation: the convergence of multiple signalling pathways on ROS production?" Nature Reviews Immunology, vol. 10, no. 3, pp. 210$215,2010$.

[80] B. Prajapati, P. K. Jena, P. Rajput, K. Purandhar, and S. Seshadri, "Understanding and modulating the Toll Like Receptors (TLRs) and NOD Like Receptors (NLRs) cross talk in type 2 diabetes," Current Diabetes Reviews, vol. 10, no. 3, pp. 190-200, 2014.

[81] J. Wang, J. Qin, Y. Li et al., "A metagenome-wide association study of gut microbiota in type 2 diabetes," Nature, vol. 490, no. 7418, pp. 55-60, 2012.

[82] C. M. Larsen, M. Faulenbach, A. Vaag et al., "Interleukin1-receptor antagonist in type 2 diabetes mellitus," The New England Journal of Medicine, vol. 356, no. 15, pp. 1517-1526, 2007.

[83] P. M. Ridker, C. P. Howard, V. Walter et al., "Effects of interleukin- $1 \beta$ inhibition with canakinumab on hemoglobin Alc, lipids, C-reactive protein, interleukin-6, and fibrinogen a phase IIb randomized, placebo-controlled trial," Circulation, vol. 126, no. 23, pp. 2739-2748, 2012.

[84] J. Hensen, C. P. Howard, V. Walter, and T. Thuren, "Impact of interleukin-1 $\beta$ antibody (canakinumab) on glycaemic indicators in patients with type 2 diabetes mellitus: results of secondary endpoints from a randomized, placebo-controlled trial," Diabetes and Metabolism, vol. 39, no. 6, pp. 524-531, 2013.

[85] C. Herder, E. Dalmas, M. Böni-Schnetzler, and M. Y. Donath, "The IL-1 pathway in type 2 diabetes and cardiovascular complications," Trends in Endocrinology and Metabolism, vol. 26, no. 10, pp. 551-563, 2015. 
[86] M. Stepanova, E. Rodriguez, A. Birerdinc, and A. Baranova, "Age-independent rise of inflammatory scores may contribute to accelerated aging in multi-morbidity," Oncotarget, vol. 6, no. 3, pp. 1414-1421, 2015.

[87] F. Prattichizzo, A. Giuliani, R. Recchioni et al., "Anti-TNF- $\alpha$ treatment modulates SASP and SASP-related microRNAs in endothelial cells and in circulating angiogenic cells," Oncotarget, vol. 7, no. 11, pp. 11945-11958, 2016.

[88] P. M. Ridker, "From C-reactive protein to interleukin-6 to interleukin-1: moving upstream to identify novel targets for atheroprotection," Circulation Research, vol. 118, no. 1, pp. 145156, 2016.

[89] A. Ceriello, "The emerging challenge in diabetes: the 'metabolic memory"' Vascular Pharmacology, vol. 57, no. 5-6, pp. 133-138, 2012.

[90] N. D. Russell and M. E. Cooper, "50 years forward: mechanisms of hyperglycaemia-driven diabetic complications," Diabetologia, vol. 58, no. 8, pp. 1708-1714, 2015.

[91] R. R. Holman, S. K. Paul, M. A. Bethel, D. R. Matthews, and H. A. W. Neil, "10-Year follow-up of intensive glucose control in type 2 diabetes," The New England Journal of Medicine, vol. 359, no. 15, pp. 1577-1589, 2008.

[92] A. A. Eid, B. M. Ford, B. Bhandary et al., "Mammalian target of rapamycin regulates Nox4-mediated podocyte depletion in diabetic renal injury," Diabetes, vol. 62, no. 8, pp. 2935-2947, 2013.

[93] C. Zhao, D. Yasumura, X. Li et al., "mTOR-mediated dedifferentiation of the retinal pigment epithelium initiates photoreceptor degeneration in mice," Journal of Clinical Investigation, vol. 121, no. 1, pp. 369-383, 2011.

[94] M. V. Blagosklonny, "TOR-centric view on insulin resistance and diabetic complications: perspective for endocrinologists and gerontologists," Cell Death and Disease, vol. 4, no. 12, article e964, 2013.

[95] F. Forouzandeh, G. Salazar, N. Patrushev et al., "Metformin beyond diabetes: pleiotropic benefits of metformin in attenuation of atherosclerosis," Journal of the American Heart Association, vol. 3, no. 6, Article ID e001202, 2014.

[96] O. Moiseeva, X. Deschênes-Simard, E. St-Germain et al., "Metformin inhibits the senescence-associated secretory phenotype by interfering with IKK/NF- $\kappa$ B activation," Aging Cell, vol. 12, no. 3, pp. 489-498, 2013.

[97] J. R. Dörr, Y. Yu, M. Milanovic et al., "Synthetic lethal metabolic targeting of cellular senescence in cancer therapy," Nature, vol. 501, no. 7467, pp. 421-425, 2013.

[98] F. Olivieri, R. Lazzarini, L. Babini et al., "Anti-inflammatory effect of ubiquinol-10 on young and senescent endothelial cells via miR-146a modulation," Free Radical Biology and Medicine, vol. 63, pp. 410-420, 2013.

[99] J. Haendeler, J. Hoffmann, J. F. Diehl et al., "Antioxidants inhibit nuclear export of telomerase reverse transcriptase and delay replicative senescence of endothelial cells," Circulation Research, vol. 94, no. 6, pp. 768-775, 2004.

[100] Y. Hong, M. Quintero, N. M. Frakich, E. Trivier, and J. D. Erusalimsky, "Evidence against the involvement of nitric oxide in the modulation of telomerase activity or replicative capacity of human endothelial cells," Experimental Gerontology, vol. 42, no. 9, pp. 904-910, 2007.

[101] H. D. Sesso, J. E. Buring, W. G. Christen et al., "Vitamins E and $\mathrm{C}$ in the prevention of cardiovascular disease in men: the Physicians' Health Study II randomized controlled trial," The
Journal of the American Medical Association, vol. 300, no. 18, pp. 2123-2133, 2008.

[102] D. Gems and L. Partridge, "Stress-response hormesis and aging: 'that which does not kill us makes us stronger,' Cell Metabolism, vol. 7, no. 3, pp. 200-203, 2008.

[103] S. V. Brodsky and M. S. Goligorsky, "Endothelium under stress: local and systemic messages," Seminars in Nephrology, vol. 32, no. 2, pp. 192-198, 2012.

[104] K. Maiese, "Yin yang: a balancing act for oxidative stress," Oxidative Medicine and Cellular Longevity, vol. 3, no. 4, article 227, 2010.

[105] A. Rosso, A. Balsamo, R. Gambino et al., "p53 mediates the accelerated onset of senescence of endothelial progenitor cells in diabetes," The Journal of Biological Chemistry, vol. 281, no. 7, pp. 4339-4347, 2006.

[106] S. V. Brodsky, O. Gealekman, J. Chen et al., "Prevention and reversal of premature endothelial cell senescence and vasculopathy in obesity-induced diabetes by ebselen," Circulation Research, vol. 94, no. 3, pp. 377-384, 2004.

[107] A. Ceriello, A. Novials, E. Ortega et al., "Evidence that hyperglycemia after recovery from hypoglycemia worsens endothelial function and increases oxidative stress and inflammation in healthy control subjects and subjects with type 1 diabetes," Diabetes, vol. 61, no. 11, pp. 2993-2997, 2012.

[108] A. Ceriello and R. Testa, "Antioxidant anti-inflammatory treatment in type 2 diabetes," Diabetes Care, vol. 32, supplement 2, pp. S232-236, 2009.

[109] K. Maiese, "New insights for oxidative stress and diabetes mellitus," Oxidative Medicine and Cellular Longevity, vol. 2015, Article ID 875961, 17 pages, 2015.

[110] J. A. Mattison, M. Wang, M. Bernier et al., "Resveratrol prevents high fat/sucrose diet-induced central arterial wall inflammation and stiffening in nonhuman primates," Cell Metabolism, vol. 20, no. 1, pp. 183-190, 2014.

[111] Y. Jimenez-Gomez, J. A. Mattison, K. J. Pearson et al., "Resveratrol improves adipose insulin signaling and reduces the inflammatory response in adipose tissue of rhesus monkeys on highfat, high-sugar diet," Cell Metabolism, vol. 18, no. 4, pp. 533-545, 2013.

[112] M. Cnop, F. Foufelle, and L. A. Velloso, "Endoplasmic reticulum stress, obesity and diabetes," Trends in Molecular Medicine, vol. 18, no. 1, pp. 59-68, 2012.

[113] M. Flamment, E. Hajduch, P. Ferré, and F. Foufelle, "New insights into ER stress-induced insulin resistance," Trends in Endocrinology and Metabolism, vol. 23, no. 8, pp. 381-390, 2012.

[114] M. F. Gregor, L. Yang, E. Fabbrini et al., "Endoplasmic reticulum stress is reduced in tissues of obese subjects after weight loss," Diabetes, vol. 58, no. 3, pp. 693-700, 2009.

[115] S. B. Cullinan and J. A. Diehl, "Coordination of ER and oxidative stress signaling: the PERK/Nrf2 signaling pathway," International Journal of Biochemistry and Cell Biology, vol. 38, no. 3, pp. 317-332, 2006.

[116] G. S. Hotamisligil, "Endoplasmic reticulum stress and atherosclerosis," Nature Medicine, vol. 16, no. 4, pp. 396-399, 2010.

[117] G. Pujadas, V. De Nigris, L. La Sala, R. Testa, S. Genovese, and A. Ceriello, "The pivotal role of high glucose-induced overexpression of $\mathrm{PKC} \beta$ in the appearance of glucagon-like peptide-1 resistance in endothelial cells," Endocrine, 2015.

[118] R. Cunard and K. Sharma, "The endoplasmic reticulum stress response and diabetic kidney disease," American Journal of 
Physiology. Renal Physiology, vol. 300, no. 5, pp. F1054-F1061, 2011.

[119] C. Denoyelle, G. Abou-Rjaily, V. Bezrookove et al., "Antioncogenic role of the endoplasmic reticulum differentially activated by mutations in the MAPK pathway," Nature Cell Biology, vol. 8, no. 10, pp. 1053-1063, 2006.

[120] B. Zhu, C. H. Ferry, L. K. Markell et al., “The nuclear receptor peroxisome proliferator-activated receptor- $\beta / \delta \quad(\operatorname{PPAR} \beta / \delta)$ promotes oncogene-induced cellular senescence through repression of endoplasmic reticulum stress," The Journal of Biological Chemistry, vol. 289, no. 29, pp. 20102-20119, 2014.

[121] E. J. Fernandez and E. Lolis, "Structure, function, and inhibition of chemokines," Annual Review of Pharmacology and Toxicology, vol. 42, pp. 469-499, 2002.

[122] A. Higa and E. Chevet, "Redox signaling loops in the unfolded protein response," Cellular Signalling, vol. 24, no. 8, pp. 15481555, 2012.

[123] R. Kodama, M. Kato, S. Furuta et al., "ROS-generating oxidases Noxl and Nox 4 contribute to oncogenic Ras-induced premature senescence," Genes to Cells, vol. 18, no. 1, pp. 32-41, 2013.

[124] J. Hollien, J. H. Lin, H. Li, N. Stevens, P. Walter, and J. S. Weissman, "Regulated Irel-dependent decay of messenger RNAs in mammalian cells," The Journal of Cell Biology, vol. 186, no. 3, pp. 323-331, 2009.

[125] B. P. Chen, M. Li, and A. Asaithamby, "New insights into the roles of ATM and DNA-PKcs in the cellular response to oxidative stress," Cancer Letters, vol. 327, no. 1-2, pp. 103-110, 2012. 


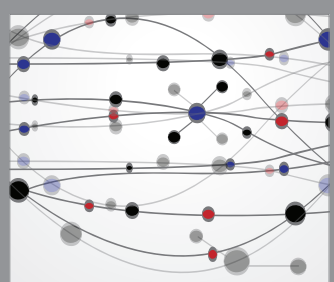

The Scientific World Journal
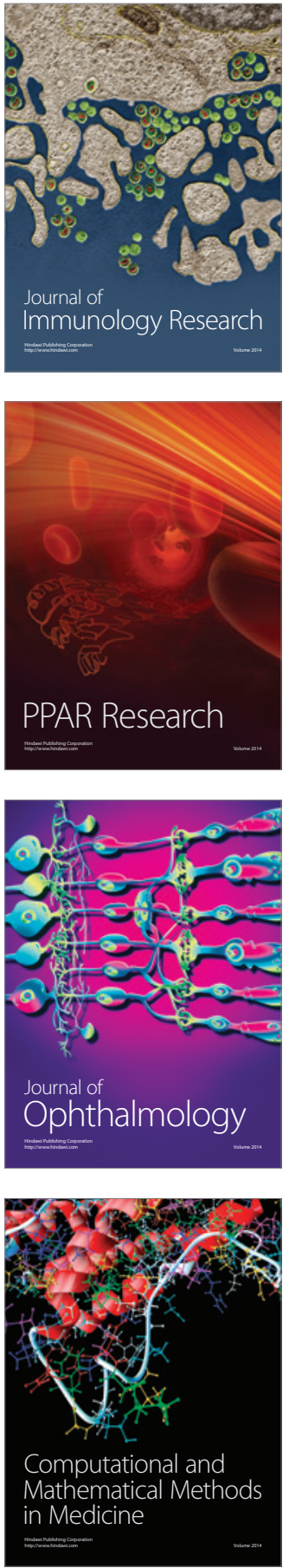

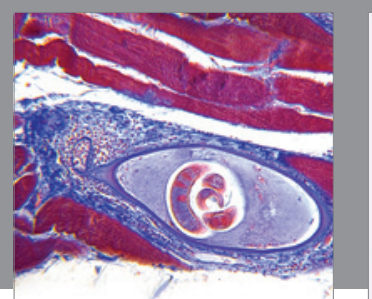

Gastroenterology Research and Practice

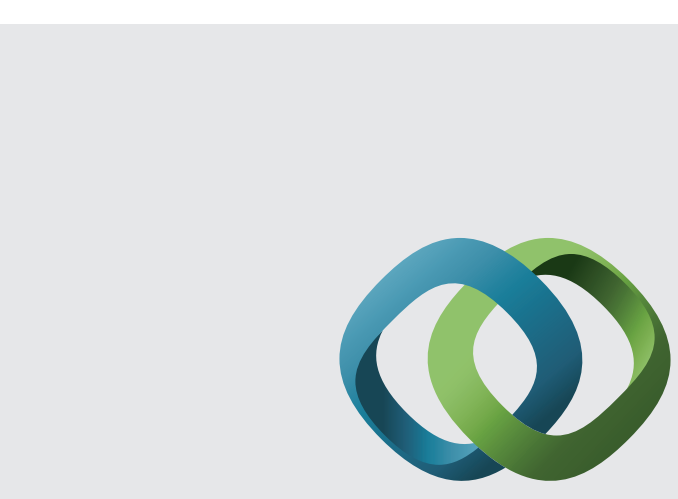

\section{Hindawi}

Submit your manuscripts at

http://www.hindawi.com
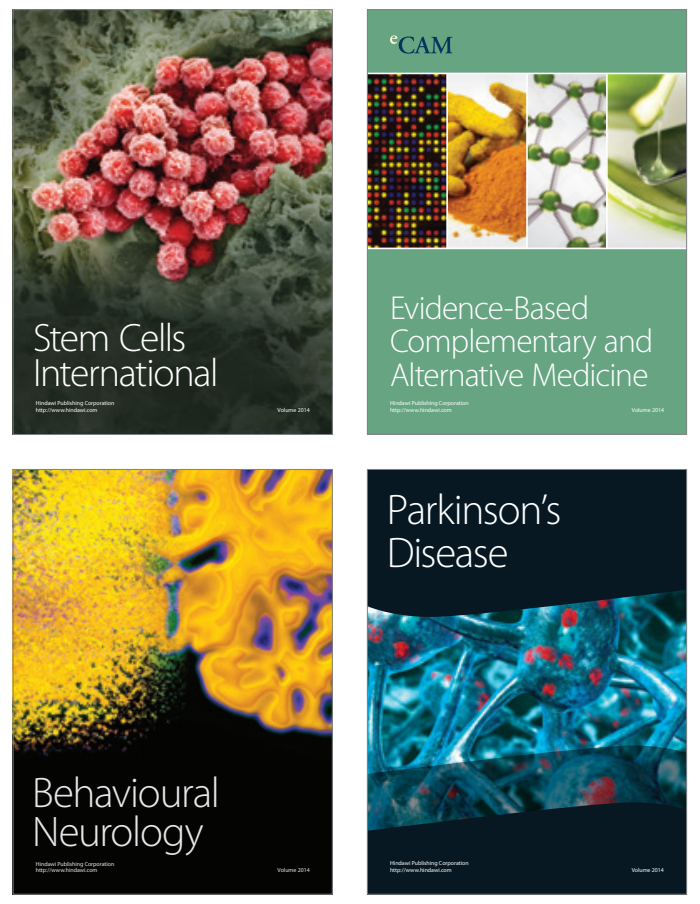
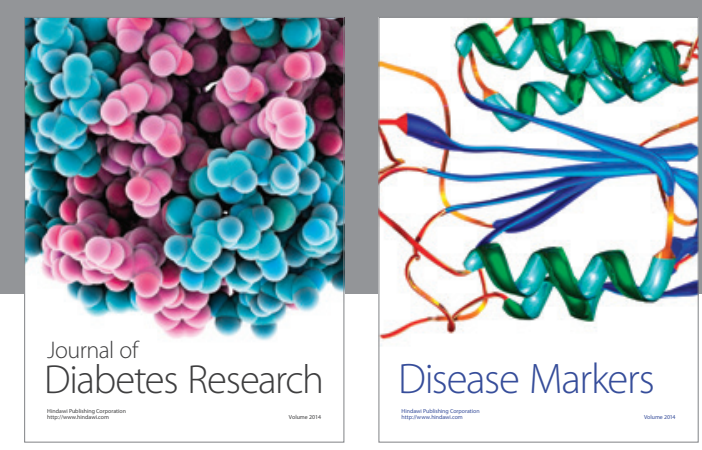

Disease Markers
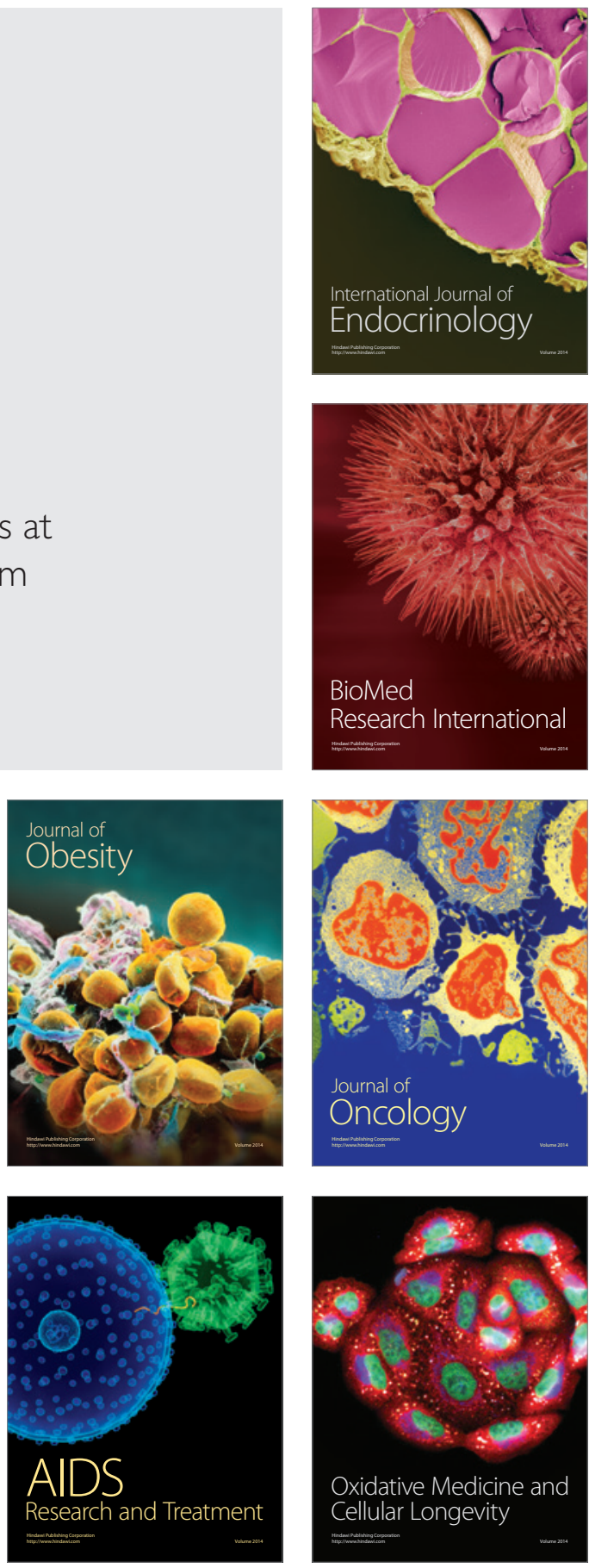